\title{
Glucose-6-phosphate dehydrogenase blockade potentiates tyrosine kinase inhibitor effect on breast cancer cells through autophagy perturbation
}

Luigi Mele ${ }^{1 \dagger}$, Marcella la Noce ${ }^{1 \dagger}$, Francesca Paino $^{2 \dagger}$, Tarik Regad ${ }^{3,4}$, Sarah Wagner ${ }^{4}$, Davide Liccardo ${ }^{1}$, Gianpaolo Papaccio ${ }^{1 *}$, Angela Lombardi ${ }^{1}$, Michele Caraglia ${ }^{3,5}$, Virginia Tirino ${ }^{1+}$, Vincenzo Desiderio ${ }^{1 *+}$ (D) and Federica Papaccio ${ }^{1 \dagger}$

\begin{abstract}
Background: Glucose-6-phospate dehydrogenase (G6PD) is the limiting enzyme of the pentose phosphate pathway (PPP) correlated to cancer progression and drug resistance. We previously showed that G6PD inhibition leads to Endoplasmic Reticulum (ER) stress often associated to autophagy deregulation. The latter can be induced by target-based agents such as Lapatinib, an anti-HER2 tyrosine kinase inhibitor (TKI) largely used in breast cancer treatment.

Methods: Here we investigate whether G6PD inhibition causes autophagy alteration, which can potentiate Lapatinib effect on cancer cells. Immunofluorescence and flow cytometry for LC3B and lysosomes tracker were used to study autophagy in cells treated with lapatinib and/or G6PD inhibitors (polydatin). Immunoblots for LC3B and p62 were performed to confirm autophagy flux analyses together with puncta and colocalization studies. We generated a cell line overexpressing G6PD and performed synergism studies on cell growth inhibition induced by Lapatinib and Polydatin using the median effect by Chou-Talay. Synergism studies were additionally validated with apoptosis analysis by annexin V/PI staining in the presence or absence of autophagy blockers.

Results: We found that the inhibition of G6PD induced endoplasmic reticulum stress, which was responsible for the deregulation of autophagy flux. Indeed, G6PD blockade caused a consistent increase of autophagosomes formation independently from mTOR status. Cells engineered to overexpress G6PD became resilient to autophagy and resistant to lapatinib. On the other hand, G6PD inhibition synergistically increased lapatinib-induced cytotoxic effect on cancer cells, while autophagy blockade abolished this effect. Finally, in silico studies showed a significant correlation between G6PD expression and tumour relapse/resistance in patients.
\end{abstract}

Conclusions: These results point out that autophagy and PPP are crucial players in TKI resistance, and highlight a peculiar vulnerability of breast cancer cells, where impairment of metabolic pathways and autophagy could be used to reinforce TKI efficacy in cancer treatment.

Keywords: Pentose phosphate pathway, Autophagy, Lapatinib, ER stress, TKI, Breast cancer, Polydatin

\footnotetext{
* Correspondence: gianpaolo.papaccio@unicampania.it;

vincenzo.desiderio@unicampania.it

tLuigi Mele, Marcella la Noce and Francesca Paino contributed equally to this

work

${ }^{+}$Virginia Tirino, Vincenzo Desiderio and Federica Papaccio are co-last authors

'Department of Experimental Medicine, University of Campania "Luigi

Vanvitelli", Via Luciano Armanni, 5, 80138 Napoli, Naples, Italy

Full list of author information is available at the end of the article
}

C The Author(s). 2019 Open Access This article is distributed under the terms of the Creative Commons Attribution 4.0 International License (http://creativecommons.org/licenses/by/4.0/), which permits unrestricted use, distribution, and reproduction in any medium, provided you give appropriate credit to the original author(s) and the source, provide a link to the Creative Commons license, and indicate if changes were made. The Creative Commons Public Domain Dedication waiver (http://creativecommons.org/publicdomain/zero/1.0/) applies to the data made available in this article, unless otherwise stated. 


\section{Background}

In recent years, metabolic deregulations have been studied as prognostic factors for tumours and as potential targets for innovative treatments [1-4]. Pentose phosphate pathway (PPP), a cytoplasmic metabolic process parallel to glycolysis, synthesizes the nucleotide precursor ribose-5-phosphate and produces NADPH, the reduced form of $\mathrm{NADP}^{+}$(the nicotinamide adenine dinucleotide phosphate), that is an essential cofactor for the synthesis of lipids and maintenance of redox balance of the cell $[5,6]$. NADPH counteracts oxidative stress produced in highly metabolizing cancer cells in form of reactive oxygen species (ROS). Glucose-6-phosphate dehydrogenase (G6PD) is the key enzyme of the PPP, it is often over-expressed in several types of cancer such as breast cancer, oesophageal carcinoma, renal cancer and is correlated to worse prognosis [7-10]. Indeed, some of the main oncogenes and tumour suppressors such as p53 and K-Ras, can directly regulate the enzymes involved in this pathway $[6,11,12]$. Several studies showed that the inhibition of G6PD may result in the development of therapeutic strategies against tumour growth and metastasis [13-15].

Breast cancer is the most common type of cancer in women and is associated with high mortality that is due to tumour aggressiveness and drug resistance. This cancer is classified according to the expression of oestrogen receptor (ERec), progesterone receptor (PR), and/or human epidermal growth factor receptor 2 (HER-2). The expression of these receptors determines the phenotype of the breast cancer and thus defines the therapeutic strategy to follow $[16,17]$. Lapatinib, an orally administered small-molecule, is a dual tyrosine kinase inhibitor (TKI) that targets both HER-2 receptor and the epidermal growth factor receptor (EGFR), and which has been widely used for the treatment of breast cancer [18]. This inhibitor induces apoptosis and autophagy in cancer cells. However, resistance to lapatinib treatment is also observed. The resistance can be caused by alterations in autophagosome and autolysosome proteins, suggesting a potential role of autophagy [19-21]. Furthermore, the increase of the antioxidant metabolic pathway has been associated with TKI resistance in cancer. This suggests that the strategies based on the targeting of antioxidant metabolic pathways may improve the efficiency of TKI-based therapy [22]. In this study, we investigated the interplay between G6PD, ER stress and autophagy, and highlighted new possible strategies to improve the effect of TKIs in treatment of breast cancer overcoming drug resistance.

\section{Materials and methods}

\section{Chemicals, cell culture and in vitro treatment}

All chemicals were purchased from Sigma-Aldrich (St. Louis, USA) unless otherwise specified. Trans-polydatin, with a purity grade higher than $99 \%$, was been supplied by Ghimas spa (Casalecchio, Bologna, Italy). Selective inhibitors of IRE1 $\alpha(4 \mu 8 C)$ and PERK (GSK 2606414) were obtained from Tocris Bioscience (Bristol, United Kingdom). Lapatinib (Tyverb ${ }^{\odot}$ ) was obtained from GSK (Verona, Italy). MCF7 cells were purchased from ATCC. Cells were cultured in DMEM (Gibco, NY, USA) supplemented with $2 \mathrm{mM}$ glutamine, $100 \mathrm{IU} / \mathrm{mL}$ penicillin, $100 \mu \mathrm{g} / \mathrm{mL}$ streptomycin (Invitrogen, Carlsbad, CA), and $10 \%$ heat-inactivated foetal bovine serum (FBS) (Gibco, NY, USA) at $37^{\circ} \mathrm{C}$ in a humidified atmosphere under $5 \%$ $\mathrm{CO} 2$. All cell lines were kept mycoplasma free, checking was performed every three months.

\section{Cell viability assay}

Cell viability was measured by the colorimetric 3-(4,5dimethyl-2-thiazolyl)-2,5-diphenyltetrazolium bromide (MTT) assay. Cells were seeded in 96-well plates at a density of $10^{4}$ cells per well, then they were treated with $100 \mu \mathrm{L}$ of $1 \mathrm{mg} / \mathrm{mL}$ MTT (Sigma) in DMEM medium containing $10 \%$ foetal bovine serum for $4 \mathrm{~h}$ at $37^{\circ} \mathrm{C}$. The medium was then replaced with $200 \mu \mathrm{L}$ of DMSO and shaken for $15 \mathrm{~min}$, then absorbance at $540 \mathrm{~nm}$ was measured using a microplate ELISA reader with DMSO used as the blank. To quantify the synergistic or antagonist effect of the drugs combinations, CompuSyn software was used [23].

\section{Immunofluorescence staining}

After $24 \mathrm{~h}$ treatment with PD at various concentrations or $30 \mu \mathrm{M}$ chloroquine (autophagy positive control), cells were washed in PBS and fixed with $4 \%$ paraformaldehyde (PFA) solution and permeabilized with $0.1 \%$ TRITON -X/PBS solution, then was performed a blocking in $1 \%$ BSA for 1 hat RT. Cells were incubated with LC3B antibody (Cell Signalling, USA) and Anti-SQSTM1/p62 (ab56416, Abcam, Cambridge, UK) in PBS for $30 \mathrm{~min}$. Secondary antibodies were added after a PBS wash in the same conditions. Cells were incubated in a 1:500 solution of $10 \mathrm{mg} / \mathrm{mL}$ Hoechst (Invitrogen) in PBS for 10 min in the dark. To stain endoplasmic reticulum cells were incubated with $200 \mathrm{nM}$ ER-Tracker Blue-White DPX in PBS solution for $20 \mathrm{~min}$ at $37^{\circ} \mathrm{C}$. For positive control cells were exposed for $16 \mathrm{~h}$ to $5 \mu \mathrm{g} / \mathrm{mL}$ tunicamycin. Images were collected under a fluorescence microscope (EVOS FL Cell Imaging System, Thermo Scientific, Rockford, USA). To stain lysosomes, cells were incubated with $60 \mathrm{nM}$ LysoTracker (Thermo Fisher Scientific, USA) for $45 \mathrm{~min}$ at $37^{\circ} \mathrm{C}$. Image (Fiji plugin) software was used for the calculation of Puncta and Intensity correlation quotient (ICQ).

\section{FACS analysis}

For intracellular staining cells were fixed with Fix and Perm Reagent A (Invitrogen) for $20 \mathrm{~min}$, and then resuspended 
in Fix and Perm Reagent B (Invitrogen) for 30 min containing primary LC3B antibody (Cell Signalling, USA).

Secondary antibodies were added in Fix and Perm Reagent B (Invitrogen) in the same conditions. Apoptosis (Annexin $\mathrm{V}$ apoptosis detection kit, BD biosciences), CellROX assay (Thermo Fisher Scientific, USA), LysoTracker assay (Thermo Fisher Scientific, USA), were performed according to the manufacturer's instructions. Cells were analyzed with a FACSAria III (BD Biosciences, San Jose, CA) or a BD Accuri Cytometer (BD Biosciences, San Jose, CA). Data were analysed by FlowJo V10 software (FlowJo LLC, USA).

\section{G6PD overexpression}

p3-G6PD-t1 and negative control pCMV3-untagged-NCV (control) hygromycin-resistant plasmids were purchased from Sino Biological Inc. (Sino Biological, Beijing, China). MCF7 cells were stably transfected with Lipofectamine 3000 (Thermo Fisher Scientific, Waltham, MA USA) according to the manufacturer's instructions. Clones with upregulated expression of G6PD were selected with $100 \mu \mathrm{g} / \mathrm{mL}$ Hygromycin. Clones were screened by Western blot.

\section{Protein extraction and Western blotting}

Cells were lysed in 1x RIPA buffer $(150 \mathrm{mM} \mathrm{NaCl}, 1 \%$ NP-40, $0.5 \%$ sodium deoxycholate, $0.1 \%$ SDS, $50 \mathrm{mM}$ Tris. $\mathrm{Cl} \mathrm{pH} \mathrm{7.5)} \mathrm{plus} 1 \%$ protease inhibitor cocktail, $1 \%$ PMSF $(200 \mathrm{mM})$ and $1 \%$ sodium orthovanadate (Santa Cruz Biotechnology, USA). Lysates were clarified by centrifugation at $8000 \mathrm{x} \mathrm{g}$ for $5 \mathrm{~min}$ at $4{ }^{\circ} \mathrm{C}$ and equal amounts of protein were fractionated by SDS-PAGE and subsequently transferred onto nitrocellulose membrane, immunoblots were visualized using Supersignal ${ }^{\circ}$ West Pico Chemiluminescent substrate (Thermo Scientific, Rockford, USA). Proteins were detected with anti-Glucose 6 Phosphate Dehydrogenase (Novus Biologicals, USA); Anti-SQSTM1 / p62 (ab56416, Abcam, Cambridge, UK); anti-Phospho-Akt (Ser473) (9271, Cell Signalling, USA), anti-Akt (9272, Cell Signalling, USA); anti-phospho-mTOR (Ser2448) (2971, Cell Signalling, USA); anti-mTOR (2972, Cell Signalling, USA); anti-LC3B (2775, Cell Signalling, USA); anti- $\alpha$-Tubulin Antibody (\#2144 Cell Signalling Technology, UK); Anti-GAPDH (ab9485, Abcam, Cambridge, UK) were used for assessing loading.

\section{In silico analysis}

In silico validation was performed using gene expression profiles generated as part of the Molecular Taxonomy of Breast Cancer International Consortium (METABRIC) [24] and Pawitan [25] studies. The gene expression profiles were generated using an Illumina HT-12 v3.0 Gene Expression BeadChip (METABRIC) and an Affymetrix Human Genome U133A/U133B (Pawitan) array. The gene expression of G6PD was selected and min-max normalised across the complete patient population of both datasets. The normalised gene expression was analysed using scatter plots for the differentiation of G6PD expression across breast cancer subtypes (Her2+ enriched, Basal-like, Luminal A, Luminal B and Normal-like) and disease recurrence. Unpaired t-test was used to analyse differences between sample groups. In addition, Kaplan-Meier curves were generated presenting disease-free survival (DFS) in relation to G6PD expression. Patient groups were separated according to median expression and survival curves were analysed using Mantel-Cox test. $P$-values below 0.05 were considered to be significant $(p \leq 0.05=*$, $p \leq 0.01=* *, p \leq 0.001=* * *$ and $p \leq 0.0001=* * * *)$. Analysis was performed using GraphPad Prism 8.

\section{Results}

G6PD inhibition induces autophagy in breast cancer cells Autophagy is a biological survival mechanism that is activated in cells during stress. Activation of this pathway in cancer cells can favour or hinder cancer progression depending on cell context. In a previous work [26], we showed that the natural molecule polydatin directly inhibits G6PD by inducing reactive oxygen species (ROS) and increasing Endoplasmic Reticulum (ER) stress. Both biological effects are frequently associated with increased autophagic flux. This led us to investigate the effect of G6PD blockade on autophagy and its role in breast cancer cells response to treatment. Macroautophagy (from now on autophagy) is a cellular process that allow for orderly degradation recycling of cellular organelles. The major steps of this process include the formation of autophagosome, vesicles containing the organelles to be degraded, and successively the fusion of these with lysosome (autophagolysosomes). Autophagosomes formation involves the recruitment of LC3B (Microtubule-associated protein $1 \mathrm{~A} / 1 \mathrm{~B}$-light chain 3 ) and p62/sequestosome proteins. In order to monitor autophagy in treated cells, we performed an IF and a quantitative analysis by flow cytometry using the vital dye LysoTracker, LAMP1 and LC3B antibody. LysoTraker is a fluorescent dye for labelling and tracking acidic organelles in live cells, thus it will stain both lysosomes and autophagolysosomes. Both qualitative and quantitative analyses showed a consistent increase in autophagy following exposure to polydatin (Fig. 1a,b). Lysotracker staining showed a strong increase in acidic vesicles after treatment as assessed by measuring median fluorescence ranging from 11,603 (untreated (NT)) to 16,551 (treated with $30 \mu \mathrm{M}$ Polydatin). LC3B staining indicated the appearance of several fluorescent vesicles with a median fluorescence ranging between 41,709 (untreated) to 204,124 (treated with $30 \mu \mathrm{M}$ Polydatin). To investigate the formation of autophagic vesicles, we analysed the LC3B puncta per cells (Fig. 1c). Typically, LC3B associated to 


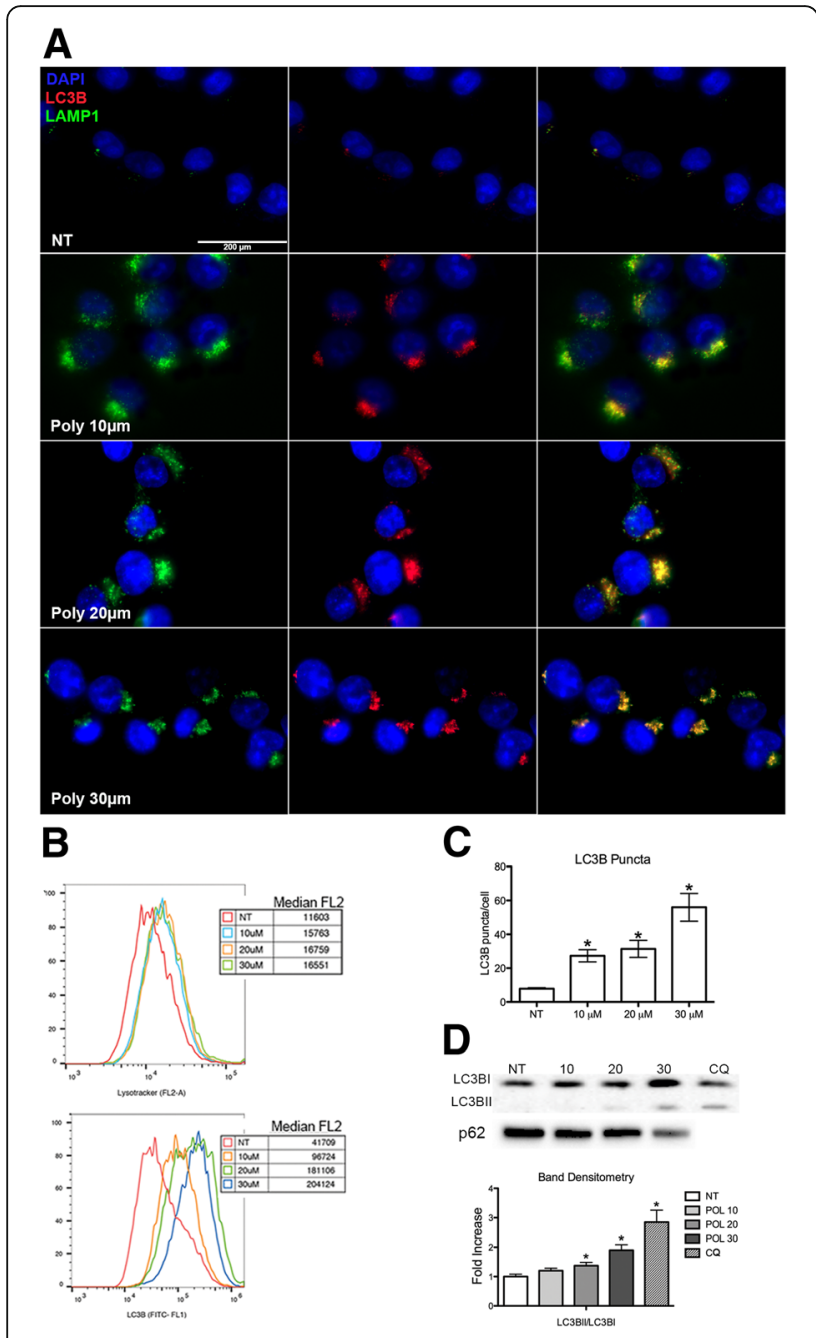

Fig. 1 G6PD inhibition induces autophagy. a Autophagy analysis on polydatin-treated cells (10-30 $\mathrm{MM})$ performed by immunofluorescence for LAMP1 (top row) and LC3B protein (lower row), $24 \mathrm{~h}$ after treatment. b Flow cytometry histograms for the Lysotraker and Lc3B, median fluorescence values are shown in the graphs. Both IF and flow cytometry show an increase of LAMP1, LysoTracker and LC3B fluorescence accordingly to polydatin concentration. c Analysis of puncta on IF for LC3B in polydatin-treated cells (10-30 $\mathrm{MM}), 24 \mathrm{~h}$ after treatment. Puncta represent single autophagosomes. $\mathbf{d}$ Immunoblot for LC3B and p62; band densitometry in polydatin treated cells (10-30 $\mu \mathrm{M}), 24 \mathrm{~h}$ after treatment. LC3BII increases accordingly to polydatin concentration while p62 decreases. $p<0.05, N=3$ (Biological replicates); error bars = SEM

autophagosomes is visualized in immunofluorescence as dots (puncta), each dot representing an autophagosome. We found a consistent increase of puncta in a Polydatin concentration-dependent manner. When is recruited on autophagosomes LC3B becomes conjugated with phosphatidylethanolamine (LC3BII) [27]. The activation of LC3BI (LC3BII) was also confirmed by immunoblotting (Fig. 1d) and band densitometry showed a significant increase in LC3BII following increasing Polydatin concentrations.
To better understand the effect of G6PD blockade on autophagy (e. g. if it increases or blocks autophagic flux with accumulation of autophagosomes), we performed an analysis of autophagic flux by co-treating the cells with chloroquine, a drug that block the fusion of autophagic vesicles and lysosomes, resulting in the blockade of autophagic flux and accumulation of autophagosomes. Thus, when a drug works by blocking the autophagy flux, its effect on autophagy markers (LC3B and p62) will be reduced or abrogated by co-treating with chloroquine; on the other hand, if a drug increases the autophagic flux its effect on autophagy markers will be increased. Indeed, cells treated with chloroquine showed a strong staining of both LysoTracker and LC3B by IF (Fig. 2 a, b) and increased protein expression of LC3BII and p62/sequestosome (Fig. 2c). When cells were co-treated with chloroquine and polydatin, a significant increase of LysoTracker staining and expression of LC3BII and p62/sequestosome were also observed (Fig. 2 a, b, c). Moreover, we calculated the Intensity correlation quotient (ICQ) on the IF using LC3B and LysoTracker double staining. The ICQ expresses the amount of co-localization between the two staining, that in this case ranged between 50 and $60 \%$ (ICQ $=0,26-0,32$ ). In all these experiments the co-treatment with polydatin and chloroquine constantly resulted in a stronger signal compared to single treatments. This suggests that polydatin induced an increase of autophagic flux.

\section{Autophagy is dependent on ER stress}

Autophagy can be induced by different mechanisms both in physiological and pathological conditions. One of the key pathways controlling autophagy activation is represented by the $\mathrm{AKT} / \mathrm{mTOR}$. TOR is a central regulator of cell growth and metabolism, and in response to nutritional and stress signals, it coordinates the balance between cell growth and autophagy. Polydatin has been suggested to act as an mTOR inhibitor and inducer of autophagy [28]. To exclude a potential and direct inhibitory effect of Polydatin on mTOR and to clarify its relation with G6PD inhibition, we performed immunoblots for the inactive and active (phosphorylated) forms of both AKT and mTOR (Fig. 3 a). This experiment does not show an inhibition of AKT and mTOR, on the contrary both AKT and mTOR expression increased while the ratio between the non-phosphorylated and the phosphorylated form did not change after treatments. These results suggest that the induction of autophagy was not driven by mTOR inhibition as reported for resveratrol but follows a different cell mechanism. Indeed, autophagy has been shown to be induced by UPR (Unfolded Protein Response), as a mechanism of degradation of misfolded proteins, which activation could be mediated by IRE1 or PERK [29-32]. In a previous work [26], we showed that G6PD inhibition induces a strong ER stress 


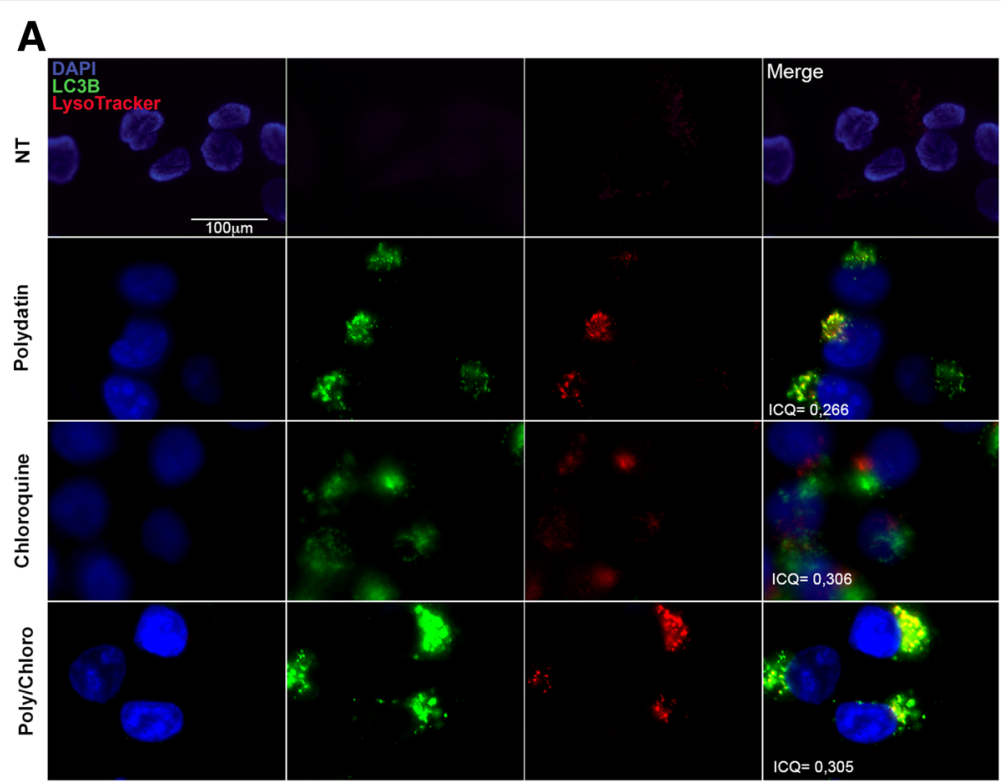

B

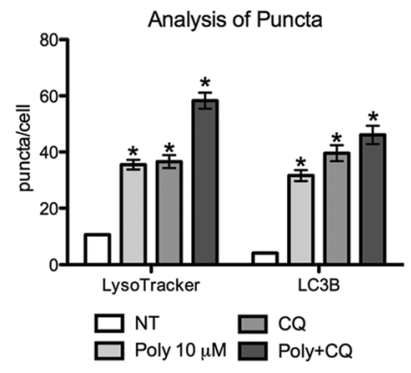

C
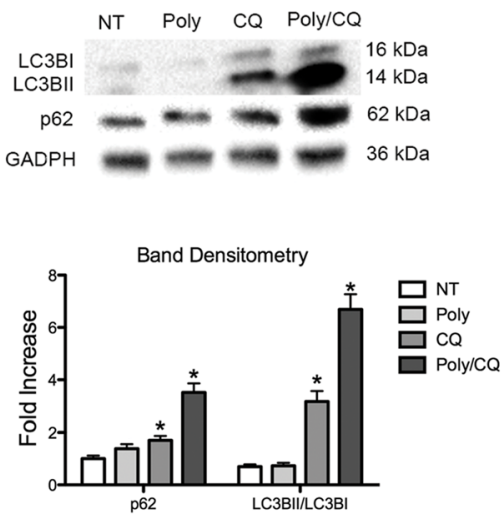

Fig. 2 Analysis of autophagy flux. a IF with Lysotracker and LC3B for autophagy flux analysis on cells treated with polydatin (10 $\mu M)$, chloroquine $(30 \mu \mathrm{M})$ and their combination. Co-treatment with chloroquine increases the fluorescence of both LysoTracker and LC3B suggesting that polydatin triggers autophagy. Intensity correlation quotient (ICQ) shows the grade of co-localization of LC3B and Lysotracker; in all three treatments a colocalization grade of about $70 \%$ was recorded. $\mathbf{b}$ Analysis of puncta for IF of LC3B and Lysotracker on cells treated with polydatin $(10 \mu \mathrm{M})$, chloroquine $(30 \mu \mathrm{M})$ and their combination. c Immunoblot with band densitometry for LC3B and P62/sequestosome on cells treated with polydatin $(10 \mu \mathrm{M})$, chloroquine $(30 \mu \mathrm{M})$ and their combination. Increase of LC3B and p62 bands in cells co-treated with polydatin and chloroquine confirms that polydatin triggers autophagic flux. ${ }^{*} p<0.05, N=3$; error bar $=95 \%$ confidence

with activation of both PERK and IRE1. Here we confirmed a strong activation of ER stress by ER TRacker staining, this vital dye stains the endoplasmic reticulum and its fluorescence was proportional to ER swelling (Fig. 3b). Inhibition of PERK or IRE1 phosphorylation by specific inhibitors reduced autophagy in polydatin-treated cells, suggesting a causative link between ER stress and autophagy (Fig. 3c).

\section{G6PD overexpression reduces autophagy and induces resistance to Lapatinib}

Lapatinib is an orally active drug for breast cancer therapy. It is a tyrosine kinase inhibitor which acts on both HER2/neu and epidermal growth factor receptor (EGFR)
[33]. Lapatinib has been shown to induce both apoptosis and autophagy in cancer cells [19-21, 34]. Based on this, we hypothesised that G6PD blockade could increase Lapatinib effect on cancer. On the other hand, G6PD overexpression is common in several cancers and correlates with aggressiveness and poor prognosis [7, 8, 35]. Thus, we generated a cell line overexpressing G6PD $\left(\mathrm{MCF} 7^{\mathrm{G} 6 \mathrm{PD}+}\right)$ [26] and compared the activation of autophagy with control cells (mock plasmid MCF7 ${ }^{\text {mock }}$ ) when treated with both polydatin and lapatinib. Lapatinib concentration was chosen from a viability assay performed on MCF7 (Fig. 5 a) to be the minimum effective concentration. As shown in Fig. 4, lapatinib induced 


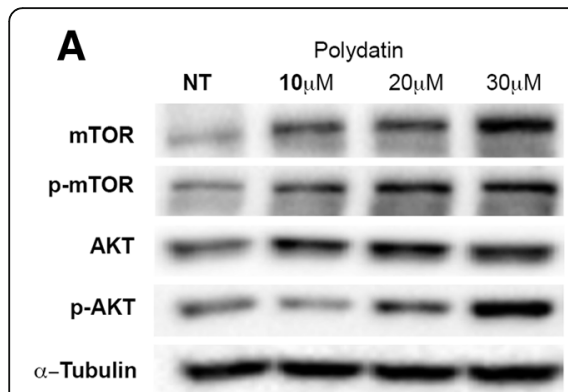

B
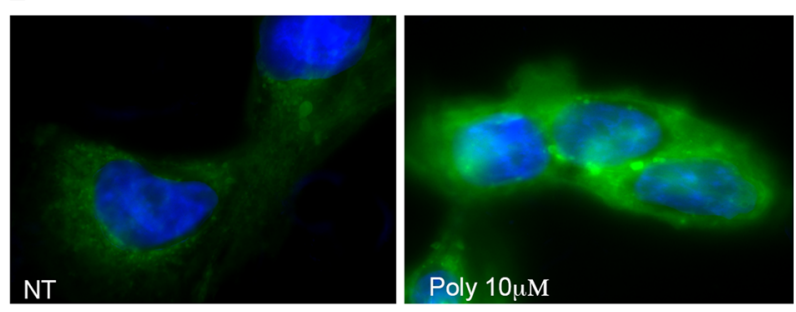

C
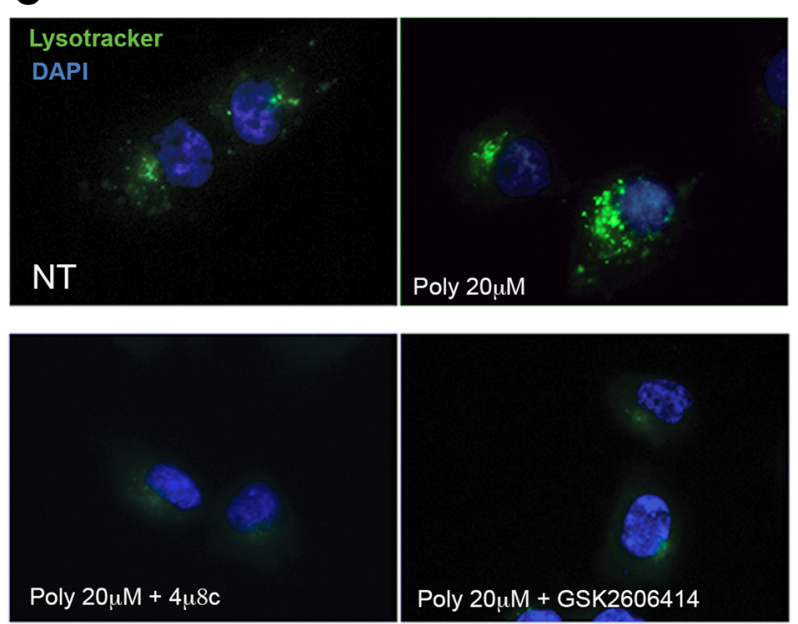
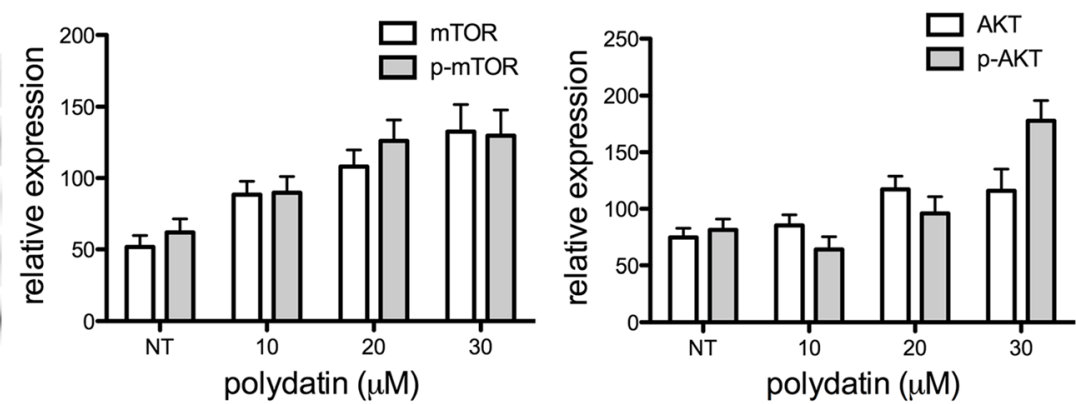
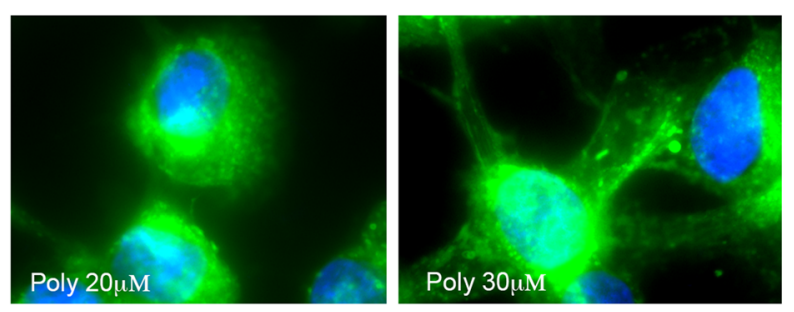

Poly $30 \mu \mathrm{M}$
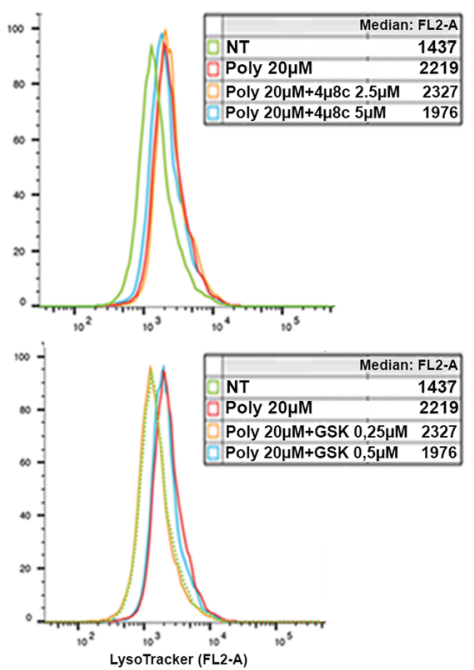

G6PD and IRE inhibitors

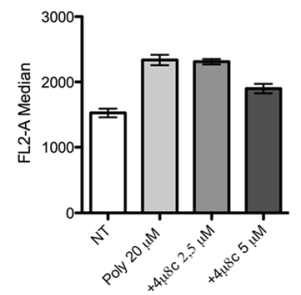

G6PD and PERK inhibitors

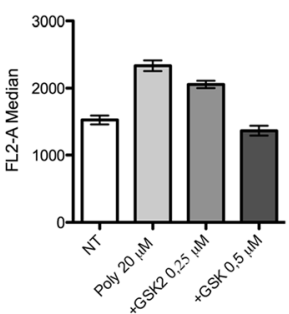

Fig. 3 Autophagy is dependent on ER stress. a Immunoblot for mTOR/p-mTOR and AKT/p-AKT with band densitometries; the total amount of both AKT and mTOR increases over polydatin treatment; however, the ratio between phosphorylated and non-phosphorylated forms does not change. b IF with ER-Tracker $24 \mathrm{~h}$ after polydatin treatment. ER Tracker stains the endoplasmic reticulum and its fluorescence is proportional to ER swelling. c IF and flow cytometry for lysotracker on cells treated with polydatin $(20 \mu \mathrm{M})$ and either IRE1 inhibitor $4 \mu 8 \mathrm{C}$ or PERK inhibitor

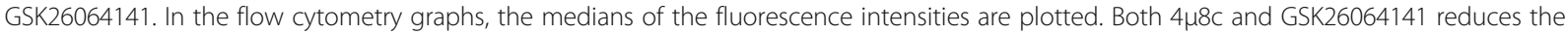
effect of polydatin on autophagy. ${ }^{*} p<0.05, N=3$ (biological replicates); error bars = SEM

autophagy in $M C F 7^{\text {mock }}$ but on much less extend on $\mathrm{MCF}^{\mathrm{G} 6 \mathrm{PD}+}$ cells as visualized with both lysotracker (Fig. 4 a) and LC3B (Fig. 4 b) for IF and flow cytometry. Interestingly, the combination lapatinib/polydatin resulted in increased activation of autophagy. In order to confirm these data, we performed an immunoblot for LAMP1, p62 and LC-3. LAMP1 increases with all treatment on MCF7 ${ }^{\text {mock }}$ confirming an increase in the lysosomal compartment. On MCF7 ${ }^{\mathrm{G} 6 \mathrm{PD}+}$ polydatin didn't produce any increase in LAMP1 while lapatinib and combo did but at much less extend than on MCF $7^{\text {mock }}$. p62 decreased on MCF7 ${ }^{\text {mock }}$ with all treatments and especially with the combo, on $\mathrm{MCF} 7^{\mathrm{G} 6 \mathrm{PD}+}$ only the combo induces a decrease. LC-3BII/LC-3BI ratio.

To show if the effect on autophagy was reflected on cell viability, we performed a viability assay and studied 


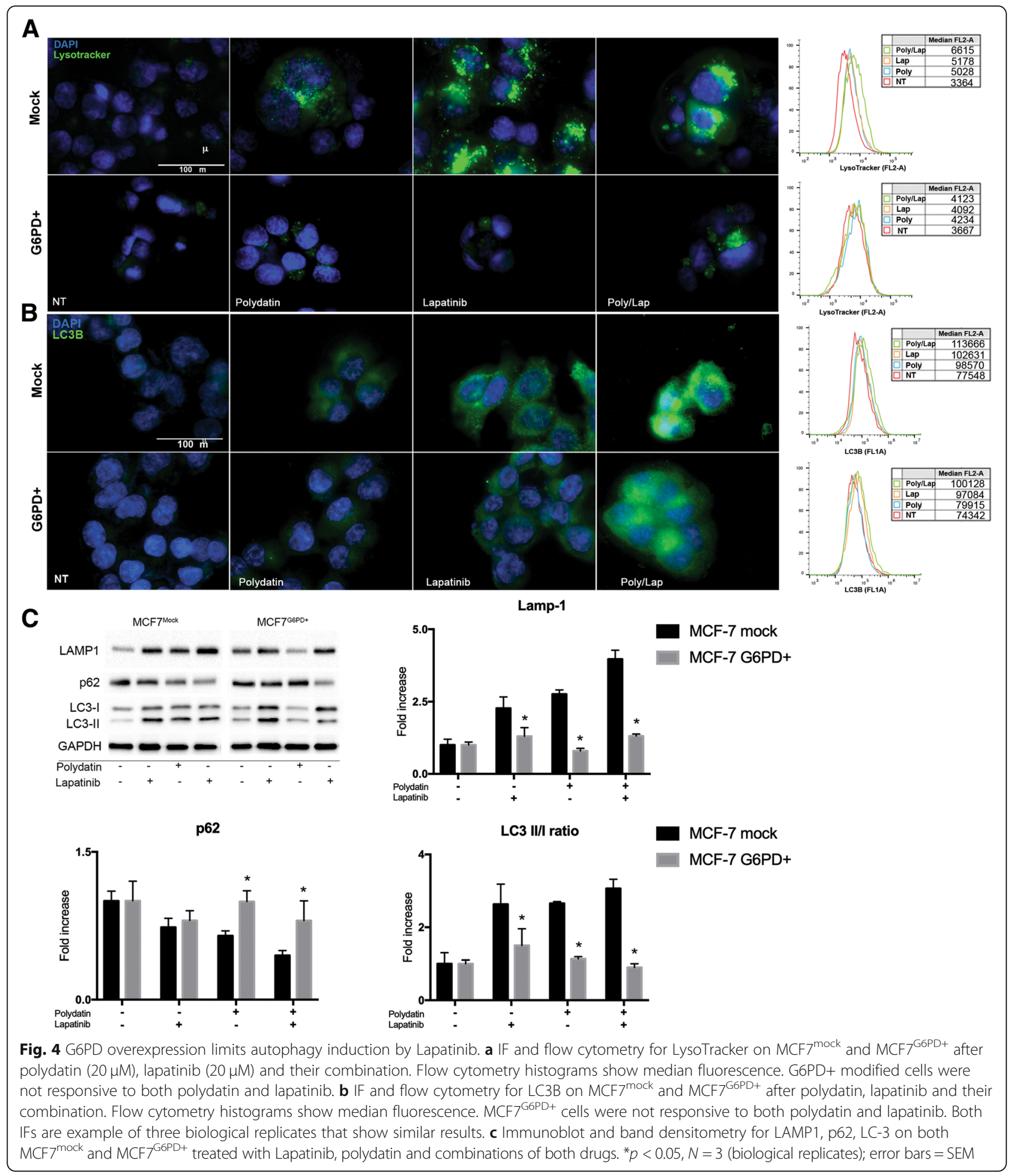

the pharmacological synergism of the combination using Chou-Talalay method [23]. The Viability assay (Fig. 5 a) showed a significant difference between $\mathrm{MCF}^{\mathrm{G} 6 \mathrm{PD}+}$ and MCF7 ${ }^{\text {mock }}$ both at $24 \mathrm{~h}$ and $48 \mathrm{~h}$ after treatment. Interestingly, lapatinib and polydatin had a synergic effect on $\mathrm{MCF}^{\text {mock }}$ but no effect was observed on $\mathrm{MCF}^{\mathrm{G} 6 \mathrm{PD}}$
${ }^{+}$cells. These data were confirmed by apoptosis analysis with Annexin V/PI and assessed by flow cytometry (Fig. 5 c, d). In order to show that the synergic effect was not limited to MCF7 cells line we performed a similar experiment on MDA231 cells line obtaining similar results (Fig. 5e). 


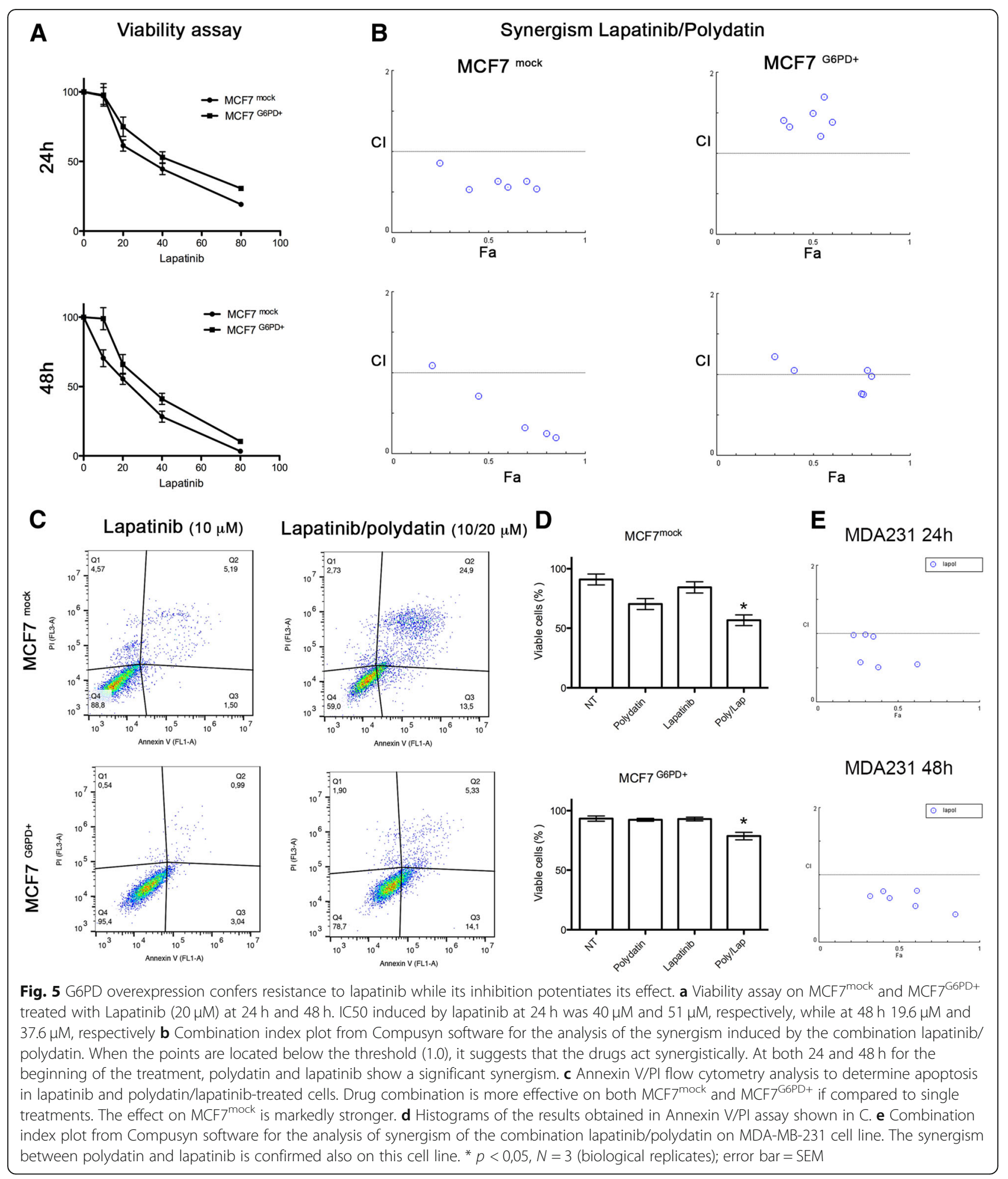

\section{Autophagy mediates cell death and synergism}

To determine if autophagy was responsible for cell death and synergistic effect between polydatin and lapatinib, we performed a study of synergism in presence of the autophagy inhibitor 3-methyladenine (3-MA). This molecule blocks autophagosome formation at earlier stages by inhibiting class III PI3K [36]. 3-MA (1 MM) concentration was chosen accordingly to the literature [36-39]. When autophagy is blocked the synergic effect seen by co-treating with polydatin and lapatinib was lost (Fig. 6 a) resulting in a 


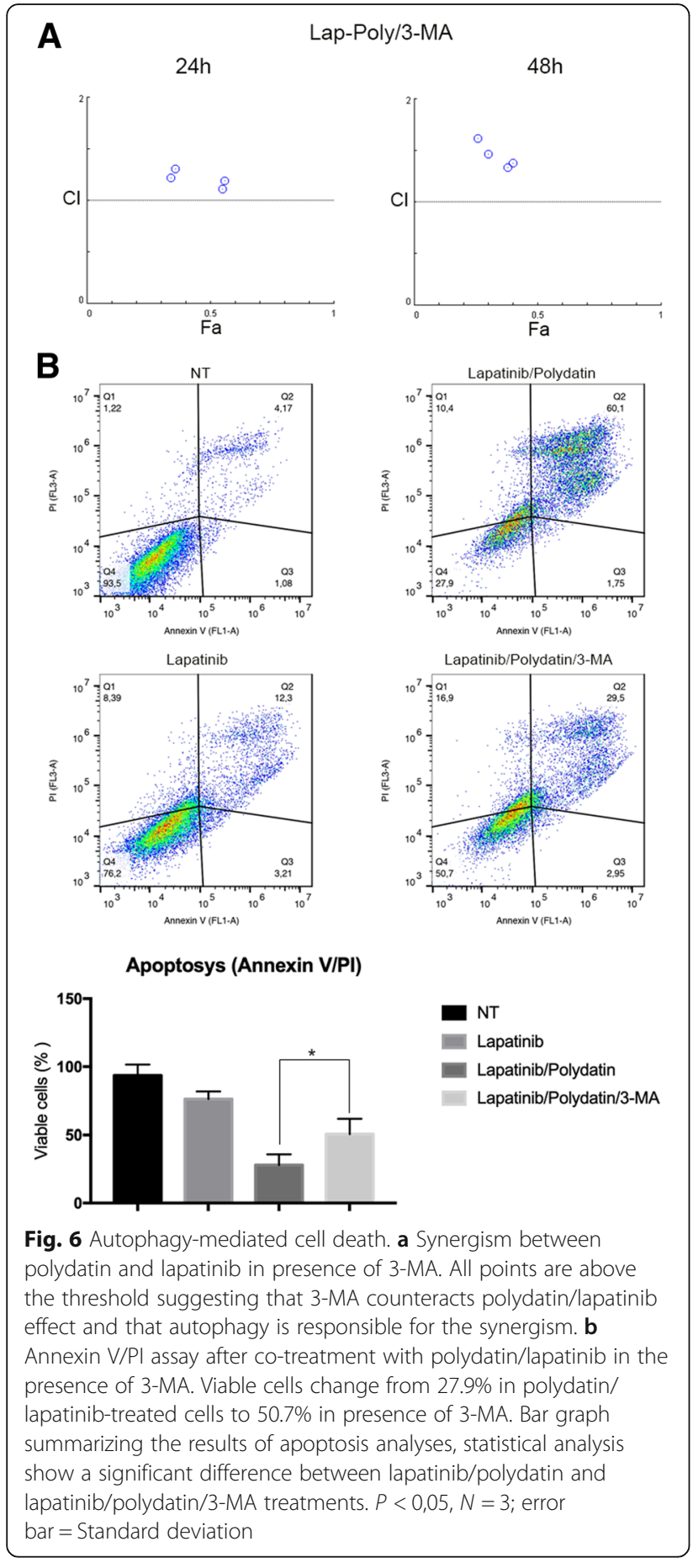

combination Index (CI) that was always higher than 1 (synergism is shown by a CI lower than 0.8 . These data were confirmed by apoptosis analysis (Fig. 6 b). In fact, lapatinib caused an about $25 \%$ reduction of viability, its combination with polydatin induced a $75 \%$ reduction, and when 3-MA was added together with polydatin and lapatinib cell viability was reduced of only $50 \%$. 3-MA counteracted the effect of lapatinib-polydatin combination, strongly suggesting that the cytotoxic effect obtained by inhibiting G6PD and Lapatinib was caused by increased autophagy.

\section{G6PD expression is correlated to disease-free survival (DFS) and recurrence in breast cancer patients}

To identify the role and impact of G6PD in breast cancer patients, an in silico analysis of two public available datasets generated through the analysis of patient-derived material was performed. The expression of G6PD across 5 breast cancer subtypes (Her2+ enriched, Basal-like, Luminal A, Luminal B and Normal-like), showed a statistically significant higher expression of G6PD in Her2+ enriched tumour material, compared to specimens derived from other breast cancer subtypes (Fig. 7a+b). Furthermore, the comparison of G6PD expression in patients with and without disease recurrence, measured at the time of diagnosis, showed a statistically significant higher expression of G6PD in patients with future disease-recurrence when compared to patients without future disease recurrence (Fig. $7 \mathrm{c}+\mathrm{d}$ ). The significance of G6PD on disease-recurrence was further supported through a Kaplan-Meier analysis of disease-free survival (DFS) time against median expression of G6PD. This analysis correlated an earlier disease-recurrence with an increased expression of G6PD at the time of diagnosis (Fig. $7 \mathrm{e}+\mathrm{f}$ ). The analysis of the METABRIC dataset has shown that patients with a lower G6PD expression have a 3 -year longer median DFS compared to patients with a high G6PD expression (Fig. 7e). Similar trends were shown for the Pawitan dataset (Fig. 7f), however both groups did not cross the $50 \%$ survival mark.

\section{Discussion}

In this manuscript, we describe for the first time that the inhibition of G6PD causes an activation of autophagic flux, which synergistically increases the cytotoxic effect of Lapatinib on breast cancer cells. Pentose phosphate pathway (PPP) is a major player in glucose catabolism that results in the production of NADPH, necessary to control redox balance, lipid metabolism and nucleotide precursors [6, 40]. G6PD is the limiting enzyme of the PPP and it is finely regulated following the cell redox state and metabolic needs. PPP activity and G6PD itself are often upregulated in cancer and are associated with aggressiveness, drug resistance and poor prognosis [7-9]. Thus, inhibition of this pathway has been pointed out as a major goal for the definition of new cancer treatments [4]. Indeed, targeting G6PD results in cancer cytotoxicity, reduction of metastases and restoration of sensitivity to drug [11, 12, 26, 35]. Nevertheless, the choice among G6PD inhibitors is very limited and debated [41]. Recently our group discovered that the natural molecule polydatin directly inhibits G6PD causing oxidative stress, endoplasmic reticulum stress and apoptosis in cancer cells [26]. Polydatin is a 

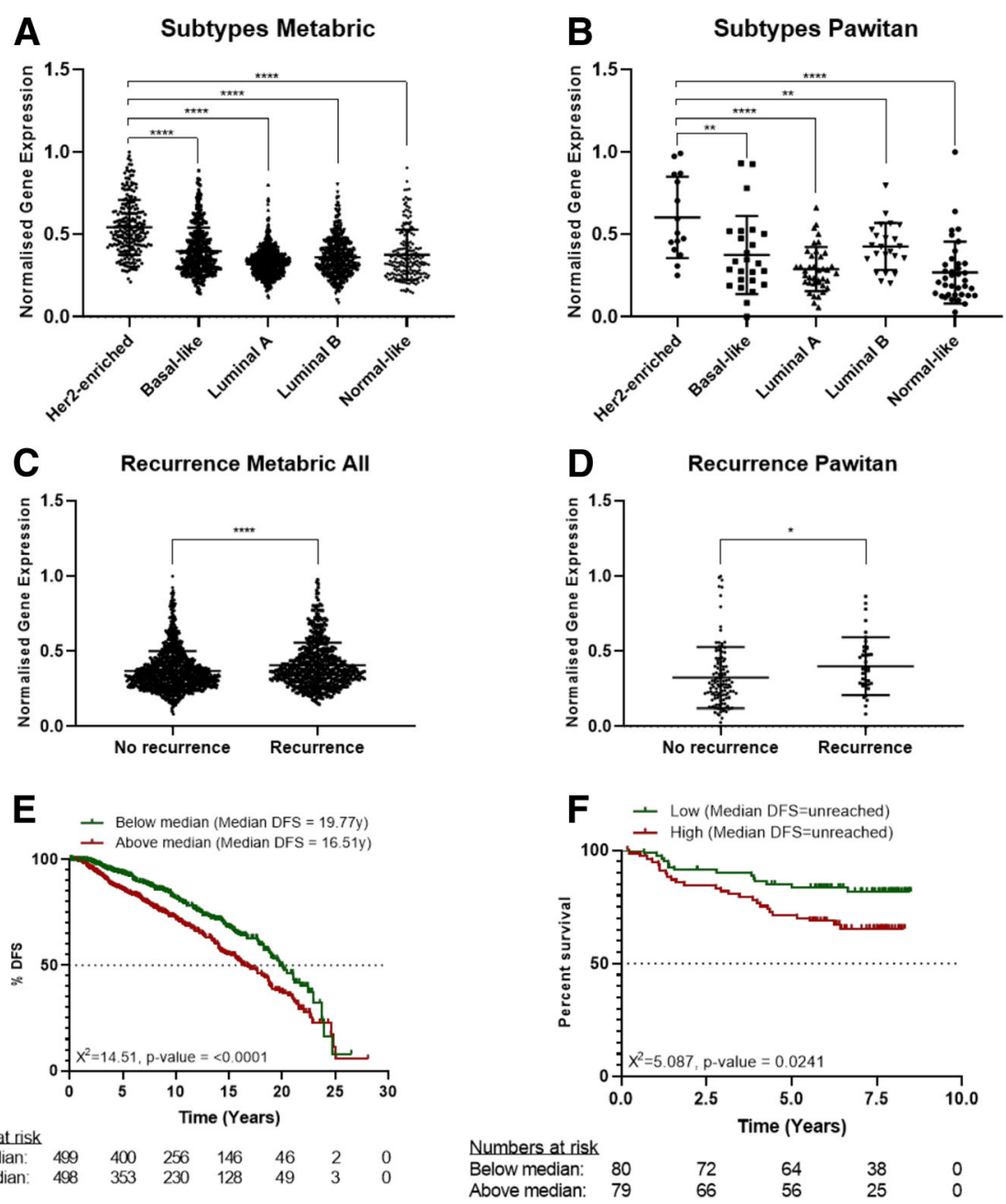

Fig. 7 G6PD is inversely correlated to DFS in breast cancer patients. a, b Scatter plot presenting the normalized gene expression of G6PD within breast cancer subtypes. Significance was tested comparing the expression in Her2+ population with other subtypes. Results highlight that the expression of G6PD is significantly higher in the Her2+ population supporting the use of a G6PD inhibitor for this group of patients. $\mathbf{c}$, $\mathbf{d}$ Scatter plot representing the normalized gene expression of G6PD in patients with disease-free survival (DFS) and patients with disease recurrence. Significant differences in the expression of G6PD is shown in the Metabric and Pawitan dataset. Both show a higher expression of G6PD in patients with disease recurrence compared to patients without disease recurrence. Note: The analysis was performed using all sample population based on the reduced numbers of Her2+ patients and the number of recurring and non-recurring patients within this selection. $\mathbf{e}$, $\mathbf{f}$ Kaplan-Meier analysis on DFS after a median split. Equal number of patients were in both groups. Significant differences in DFS are shown in the Metabric and Pawitan dataset. Both show that an increased expression of G6PD results in an earlier time of relapse. Note: The difference in the graphs of Metabric and Pawitan are based on the shorter length of follow-up of Pawitan compared to Metabric. The analysis was performed using all sample population based on the reduced numbers of Her2+ patients and the number of recurring and non-recurring patients within this selection

glucoside of resveratrol that has been studied for many years and for different pathological conditions, including inflammation and cancer [42-44]. MCF7 is most widely used breast cancer cell line for in vitro research [45]. PPP and G6PD have been proven to play an important role in their metabolism $[15,46,47]$ as well as they have been used to study lapatinib effect and resistance especially in combination with other drugs $[48,49]$. Here, we show that, G6PD inhibition induced an increase of both intracytoplasmic vesicles (puncta) that colocalize with
Lysotracker, a marker of acidic compartments, and LC3B which is associated to autophagosomes. During autophagy induction, LC3-I is converted into LC3-II with a concurrent decrease in p62. LC3B activation was confirmed also by immunoblotting.

In the study of autophagy flux, inhibition of lysosomal degradation by chloroquine or bafilomycin A causes accumulation of both LC3-II and p62, and this reflects the amount of LC3-II and p62 that would have been degraded by autophagy over the treatment period [50-52]. 
In Fig. 2c the increase of p62 and LC3B in cells co-treated with chloroquine is evident. Other studies using metabolic inhibitor show an increase in autophagic flux leading to cells death [53], this corroborate our strategy to use metabolic autophagy inducer to potentiate anticancer drug effect.

mTOR is a master regulator of cell metabolism by controlling autophagy, among other processes. Typically, when mTOR is inactive or inhibited, autophagy occurs. Therefore, most of the molecules that influence autophagy acts directly or indirectly on the mTOR pathway [54]. Resveratrol, which can be produced from polydatin degradation, has been described as mTOR inhibitor $[28,55,56]$; thus, to exclude a direct effect of polydatin on this pathway, we analysed the activation of mTOR and its upstream regulator AKT. We showed that both AKT and mTOR are not inhibited. A possible case in which autophagy is induced independently from mTOR is represented by the unfolded protein response process that is initiated following ER stress [57, 58]. Indeed, both IRE1 and PERK, which are the principal mediator of UPR, have been described to induce autophagy independently from mTOR status $[39,59]$. Our results confirmed [26] that G6PD inhibition caused ER stress and that, if UPR was inhibited by blocking IRE1 or PERK, polydatin was not still able to induce autophagy.

Tyrosine kinase inhibitors are a class of drugs that are largely used in clinical settings to treat different cancers including breast carcinoma. The work of Gregory et al. [22] pointed out that G6PD is responsible for resistance to FMS-like tyrosine kinase 3 inhibitors in acute myeloid leukaemia due to increased redox metabolism. Among TKIs that are used to treat breast cancer, lapatinib has been described to cause cancer cells cytotoxicity by inducing autophagy $[19,34]$. Therefore, we hypothesized that G6PD might play a role in modulating lapatinib effect on cancer cells. For this purpose, we created a cell line overexpressing G6PD and showed that they are resistant to autophagy induced by either lapatinib or polydatin. On the other hand, cells that received the mock plasmid showed a strong induction of autophagy, especially with the combination of the two drugs. These results were confirmed by viability analyses where the $\mathrm{MCF}^{\mathrm{G} 6 \mathrm{PD}+}$ were less sensitive to lapatinib. Moreover, the analysis of synergism between polydatin and lapatinib showed that the two molecules were highly synergistic in the MCF7 ${ }^{\text {mock }}$ at both $24 \mathrm{~h}$ and $48 \mathrm{~h}$ after treatment, while this effect was less evident or absent on the $\mathrm{MCF} 7^{\mathrm{G} 6 \mathrm{PD}+}$. These results were confirmed by Annexin V/PI analysis. Moreover, to confirm the role of autophagy in cancer cell death, we analysed both synergism and apoptosis in the presence of 3-metyladenin (3-MA), which is a widely used autophagy inhibitor $[37,60]$. Differently from chloroquine and bafilomycin
A, 3-MA inhibits autophagosomes formation at early stages. For these reasons, 3-MA has been widely used in similar experiments $[38,60]$. In these experimental conditions, synergism was completely prevented and cell viability increased from about $27 \%$ in polydatin/ lapatinib-treated cells to about $50 \%$ in the presence of 3-MA. In silico studies on two publicly available patient's databases showed a significant association between G6PD and HER2+ patients and an inverse correlation between G6PD expression and DFS. These data were on line with other reports that used different databases $[7,8]$. Possible explanation of the synergistic mechanism showed here involve autophagic cell death. Shimuzu et al. showed that Bcl-2 family of protein, which is a regulator of apoptosis, control autophagic cell death by binding to Beclin-1 and APG5 [61]. Moreover, Ros induce JNK phosphorylation can activate autophagy by interfering with the interaction between Beclin-1 and bcl-2 [62]. In addition, another mechanism that could be involved in this process is a novel programmed autophagic cells death depend on iron called ferroptosis. This involved an increase of lipid peroxides and excessive degradation of ferritin and NCOA4 [62, 63]. Future studies are necessary to understand the precise role of this mechanisms and pathways in the context described in this manuscript.

\section{Conclusion}

Taken together, our results led to the following conclusions: i. G6PD blockade caused autophagy through ER stress; ii. G6PD activity influenced lapatinib effect on cancer cells by preventing autophagy; iii. G6PD inhibitors such as polydatin might be used to increase lapatinib effect on breast cancer.

Cancer is more and more evidenced as a complex disease in which metabolic pathways play a fundamental role, either for the growth, metastases or resistance to treatments. It becomes evident that targeting these pathways is necessary to develop effective and resolute strategies. Here we provide an example in which the co-targeting of PPP and tyrosine kinase receptors lead to synergistic effects on breast cancer cells. This approach could be easily translated into clinical setting.

\footnotetext{
Abbreviations

3-MA: 3-methyladenine; Cl: Combination Index; DFS: Disease-free survival; EGFR: Epidermal growth factor receptor; ER: Endoplasmic Reticulum; ERec: Oestrogen receptor; G6PD: Glucose-6-phsphate dehydrogenase; HER2: Human epidermal growth factor receptor 2; ICQ: Intensity correlation quotient; IF: Immunofluorescence; IRE1: Inositol-requiring enzyme 1; LAMP1: Lysosomal-associated membrane protein 1; LC3B: Microtubuleassociated proteins 1A/1B light chain 3B; NADP+: Nicotinamide adenine dinucleotide phosphate; PERK: Protein kinase R (PKR)-like endoplasmic reticulum kinase; PPP: Pentose phosphate pathway; PR: Progesterone receptor; ROS: Reactive oxygen species; TKI: Tyrosine kinase inhibitor; UPR: Unfolded Protein Response; WB: Western Blot
} 


\section{Acknowledgments}

We would like to thank Dr. Paola Stiuso for kindly providing Polydatin as well as Prof Gianpietro Ravagnan for productive discussions. Moreover, we thank the association ARTOI "Associazione Ricerca Terapie Oncologiche Integrate" for supporting travel expenses to VD for the participation to the 1st Annual World Congress of Digestive Deseases-2015, Nanjing, China in December 2015. The collaboration between the University of Campania "Luigi Vanvitelli" and the Nottingham Trent University have been supported by the project CA16122 - BIONECA.

\section{Funding}

This study was supported by a grant from the "Programma VALERE 2017" of the University of Campania "Luigi Vanvitelli" (Naples, Italy).

\section{Availability of data and materials}

In this current study 2 publicly available datasets, namely METABRIC and Pawitan, were used.

The METABRIC dataset is available at the European Genome-phenome Archive under the accession number EGAS00000000098 (https://www.ebi.ac.uk/ega/ studies/EGAS00000000098).

The Pawitan dataset is available the Gene Expression Omnibus data repository under the accession number GSE1456 (https://www.ncbi.nlm.nih. gov/geo/query/acc.cgi?acc=GSE1456).

Single genes of both datasets can be accessed through CANCERTOOL (http:// web.bioinformatics.cicbiogune.es/CANCERTOOL/BCaBasicAnalyses.html).

\section{Authors' contributions}

$L M, M L N, A L$ and F. Paino performed the experiments; $L M, V T$ and VD designed the study; TR, MC, TR, SW and F. Papaccio interpreted the data; LM and VD wrote the manuscript; TR and GP proofed the manuscript. All authors read and approved the final manuscript.

\section{Ethics approval and consent to participate}

Not applicable.

\section{Consent for publication}

Not applicable.

\section{Competing interests}

The authors declare that they have no competing interests.

\section{Publisher's Note}

Springer Nature remains neutral with regard to jurisdictional claims in published maps and institutional affiliations.

\section{Author details \\ 'Department of Experimental Medicine, University of Campania "Luigi Vanvitelli", Via Luciano Armanni, 5, 80138 Napoli, Naples, Italy. ${ }^{2}$ Department of Biomedical, Surgical and Dental Sciences, University of Milan, Milan, Italy. ${ }^{3}$ Department Precision Medicine, University of Campania "Luigi Vanvitelli", 80138 Naples, Italy. ${ }^{4}$ The John van Geest Cancer Research Centre, School of Science and Technology, Nottingham Trent University, Clifton Lane, Nottingham NG11 8NS, UK. ${ }^{5}$ Molecular Oncology Laboratory, Biogem Scarl, Ariano Irpino, Avellino, Italy.}

\section{Received: 7 February 2019 Accepted: 1 April 2019}

Published online: 12 April 2019

\section{References}

1. Hirschey MD, DeBerardinis RJ, Diehl AME, Drew JE, Frezza C, Green MF, et al. Dysregulated metabolism contributes to oncogenesis. Semin Cancer Biol. 2015;35(Suppl):S129-S50.

2. Sharma AK, Konig R. Metabolic network modeling approaches for investigating the "hungry cancer". Semin Cancer Biol. 2013;23(4):227-34.

3. White E, Mehnert JM, Chan CS. Autophagy, metabolism, and Cancer. Clin Cancer Res. 2015;21(22):5037-46.

4. Jones NP, Schulze A. Targeting cancer metabolism--aiming at a tumour's sweet-spot. Drug Discov Today. 2012;17(5-6):232-41.

5. Kruger NJ, von Schaewen A. The oxidative pentose phosphate pathway: structure and organisation. Curr Opin Plant Biol. 2003;6(3):236-46.
6. Patra KC, Hay N. The pentose phosphate pathway and cancer. Trends Biochem Sci. 2014;39(8):347-54

7. Gyorffy B, Lanczky A, Eklund AC, Denkert C, Budczies J, Li Q, et al. An online survival analysis tool to rapidly assess the effect of 22,277 genes on breast cancer prognosis using microarray data of 1,809 patients. Breast Cancer Res Treat. 2010;123(3):725-31.

8. Ringner M, Fredlund E, Hakkinen J, Borg A, Staaf J. GOBO: gene expressionbased outcome for breast cancer online. PLoS One. 2011;6(3):e17911.

9. Wang X, Li X, Zhang X, Fan R, Gu H, Shi Y, et al. Glucose-6-phosphate dehydrogenase expression is correlated with poor clinical prognosis in esophageal squamous cell carcinoma. Eur J Surg Oncol. 2015;41(10):1293-9.

10. Batai K, Imler E, Pangilinan J, Bell R, Lwin A, Price E, et al. Whole-transcriptome sequencing identified gene expression signatures associated with aggressive clear cell renal cell carcinoma. Genes Cancer. 2018;9(5-6):247-56.

11. Du W, Jiang P, Mancuso A, Stonestrom A, Brewer MD, Minn AJ, et al. TAp73 enhances the pentose phosphate pathway and supports cell proliferation. Nat Cell Biol. 2013;15(8):991-1000.

12. Jiang P, Du W, Yang X. A critical role of glucose-6-phosphate dehydrogenase in TAp73-mediated cell proliferation. Cell Cycle. 2013;12(24):3720-6.

13. Xiao G, Chan LN, Klemm L, Braas D, Chen Z, Geng H, et al. B-cell-specific diversion of glucose carbon utilization reveals a unique vulnerability in $B$ cell malignancies. Cell. 2018;173(2):470-84 e18.

14. Ju HQ, Lu YX, Wu QN, Liu J, Zeng ZL, Mo HY, et al. Disrupting G6PDmediated redox homeostasis enhances chemosensitivity in colorectal cancer. Oncogene. 2017;36(45):6282-92.

15. Rao X, Duan X, Mao W, Li X, Li Z, Li Q, et al. O-GlcNAcylation of G6PD promotes the pentose phosphate pathway and tumor growth. Nat Commun. 2015;6:8468

16. Shi H, Zhang W, Zhi Q, Jiang M. Lapatinib resistance in HER2+ cancers: latest findings and new concepts on molecular mechanisms. Tumour Biol. 2016.

17. Tolaney SM, Barry WT, Dang CT, Yardley DA, Moy B, Marcom PK, et al. Adjuvant paclitaxel and trastuzumab for node-negative, HER2-positive breast cancer. N Engl J Med. 2015;372(2):134-41.

18. Voigtlaender M, Schneider-Merck T, Trepel M. Lapatinib. Recent Results Cancer Res. 2018;211:19-44.

19. Chen YJ, Chi CW, Su WC, Huang HL. Lapatinib induces autophagic cell death and inhibits growth of human hepatocellular carcinoma. Oncotarget. 2014;5(13):4845-54.

20. Zhu X, Wu L, Qiao H, Han T, Chen S, Liu X, et al. Autophagy stimulates apoptosis in HER2-overexpressing breast cancers treated by lapatinib. J Cell Biochem. 2013;114(12):2643-53.

21. Tang Y, Hamed HA, Cruickshanks N, Fisher PB, Grant S, Dent P. Obatoclax and lapatinib interact to induce toxic autophagy through NOXA. Mol Pharmacol. 2012:81(4):527-40.

22. Gregory MA, D'Alessandro A, Alvarez-Calderon F, Kim J, Nemkov T, Adane B, et al. ATM/G6PD-driven redox metabolism promotes FLT3 inhibitor resistance in acute myeloid leukemia. Proc Natl Acad Sci U S A. 2016;113(43):E6669-E78.

23. Chou TC. Drug combination studies and their synergy quantification using the Chou-Talalay method. Cancer Res. 2010;70(2):440-6.

24. Curtis C, Shah SP, Chin SF, Turashvili G, Rueda OM, Dunning MJ, et al. The genomic and transcriptomic architecture of 2,000 breast tumours reveals novel subgroups. Nature. 2012;486(7403):346-52.

25. Pawitan Y, Bjohle J, Amler L, Borg AL, Egyhazi S, Hall P, et al. Gene expression profiling spares early breast cancer patients from adjuvant therapy: derived and validated in two population-based cohorts. Breast Cancer Res. 2005;7(6):R953-64.

26. Mele L, Paino F, Papaccio F, Regad T, Boocock D, Stiuso P, et al. A new inhibitor of glucose-6-phosphate dehydrogenase blocks pentose phosphate pathway and suppresses malignant proliferation and metastasis in vivo. Cell Death Dis. 2018;9(5):572.

27. Ohsumi Y. Molecular dissection of autophagy: two ubiquitin-like systems. Nat Rev Mol Cell Biol. 2001;2(3):211-6.

28. Park D, Jeong H, Lee MN, Koh A, Kwon O, Yang YR, et al. Resveratrol induces autophagy by directly inhibiting mTOR through ATP competition. Sci Rep. 2016;6:21772.

29. Bernales S, McDonald KL, Walter P. Autophagy counterbalances endoplasmic reticulum expansion during the unfolded protein response. PLoS Biol. 2006:4(12):e423.

30. Hoyer-Hansen M, Jaattela M. Connecting endoplasmic reticulum stress to autophagy by unfolded protein response and calcium. Cell Death Differ. 2007;14(9):1576-82. 
31. Ogata M, Hino S, Saito A, Morikawa K, Kondo S, Kanemoto S, et al. Autophagy is activated for cell survival after endoplasmic reticulum stress. Mol Cell Biol. 2006;26(24):9220-31.

32. Yorimitsu T, Nair U, Yang Z, Klionsky DJ. Endoplasmic reticulum stress triggers autophagy. J Biol Chem. 2006;281(40):30299-304.

33. Cameron DA, Stein S. Drug insight: intracellular inhibitors of HER2--clinical development of lapatinib in breast cancer. Nat Clin Pract Oncol. 2008;5(9): 512-20.

34. Huang HL, Chen YC, Huang YC, Yang KC, Pan H, Shih SP, et al. Lapatinib induces autophagy, apoptosis and megakaryocytic differentiation in chronic myelogenous leukemia K562 cells. PLoS One. 2011;6(12):e29014.

35. Debeb BG, Lacerda L, Larson R, Wolfe AR, Krishnamurthy S, Reuben JM, et al. Histone deacetylase inhibitor-induced cancer stem cells exhibit high pentose phosphate pathway metabolism. Oncotarget. 2016;7(19):28329-39.

36. Heckmann BL, Yang $X$, Zhang $X$, Liu J. The autophagic inhibitor 3methyladenine potently stimulates PKA-dependent lipolysis in adipocytes. Br J Pharmacol. 2013;168(1):163-71.

37. Caro LH, Plomp PJ, Wolvetang EJ, Kerkhof C, Meijer AJ. 3-Methyladenine, an inhibitor of autophagy, has multiple effects on metabolism. Eur J Biochem. 1988;175(2):325-9.

38. Gao S, Li X, Ding X, Qi W, Yang Q. Cepharanthine induces autophagy, apoptosis and cell cycle arrest in breast Cancer cells. Cell Physiol Biochem. 2017:41(4):1633-48.

39. Wang J, Huang S, Tian R, Chen J, Gao H, Xie C, et al. The protective autophagy activated by GANT-61 in MYCN amplified neuroblastoma cells is mediated by PERK. Oncotarget. 2018;9(18):14413-27.

40. Jiang $P, D u$ W, Wu M. Regulation of the pentose phosphate pathway in cancer. Protein Cell. 2014;5(8):592-602

41. Di Monaco M, Pizzini A, Gatto V, Leonardi L, Gallo M, Brignardello E, et al. Role of glucose-6-phosphate dehydrogenase inhibition in the antiproliferative effects of dehydroepiandrosterone on human breast cancer cells. Br J Cancer. 1997;75(4):589-92.

42. De Maria S, Scognamiglio I, Lombardi A, Amodio N, Caraglia M, Carteni M, et al. Polydatin, a natural precursor of resveratrol, induces cell cycle arrest and differentiation of human colorectal Caco-2 cell. J Transl Med. 2013;11:264

43. Ravagnan G, De Filippis A, Carteni M, De Maria S, Cozza V, Petrazzuolo M, et al. Polydatin, a natural precursor of resveratrol, induces beta-defensin production and reduces inflammatory response. Inflammation. 2013;36(1):26-34.

44. Zhang Q, Feng Y, Kennedy D. Multidrug-resistant cancer cells and cancer stem cells hijack cellular systems to circumvent systemic therapies, can natural products reverse this? Cell Mol Life Sci. 2017;74(5):777-801.

45. Lee AV, Oesterreich S, Davidson NE. MCF-7 cells--changing the course of breast cancer research and care for 45 years. J Natl Cancer Inst. 2015;107(7).

46. Benito A, Polat IH, Noe V, Ciudad CJ, Marin S, Cascante M. Glucose-6phosphate dehydrogenase and transketolase modulate breast cancer cell metabolic reprogramming and correlate with poor patient outcome. Oncotarget. 2017;8(63):106693-706

47. Drabovich AP, Pavlou MP, Dimitromanolakis A, Diamandis EP. Quantitative analysis of energy metabolic pathways in MCF-7 breast cancer cells by selected reaction monitoring assay. Mol Cell Proteomics. 2012;11(8):422-34.

48. Emde AM, Maslak K, Liu H, Reles AE, Possinger K, Eucker J. Combination of fulvestrant and lapatinib in non-HER2-overexpressing and adriamycinresistant breast cancer cell lines. J Clin Oncol. 2007:25(18_suppl):14050.

49. Cruickshanks N, Tang Y, Booth L, Hamed H, Grant S, Dent P. Lapatinib and obatoclax kill breast cancer cells through reactive oxygen species-dependent endoplasmic reticulum stress. Mol Pharmacol. 2012;82(6):1217-29.

50. Chittaranjan S, Bortnik S, Gorski SM. Monitoring Autophagic flux by using lysosomal inhibitors and Western blotting of endogenous MAP 1LC3B. Cold Spring Harb Protoc. 2015:2015(8):743-50.

51. Yoshii SR, Mizushima N. Monitoring and Measuring Autophagy. Int J Mol Sci. 2017;18(9):1865.

52. Gu J, Wang $Y$, Wang $X$, Zhou $D$, Wang $X$, Zhou $M$, et al. Effect of the LnCRNA GAS5-MiR-23a-ATG3 Axis in regulating autophagy in patients with breast Cancer. Cell Physiol Biochem. 2018;48(1):194-207.

53. Jeon JY, Kim SW, Park KC, Yun M. The bifunctional autophagic flux by 2 deoxyglucose to control survival or growth of prostate cancer cells. BMC Cancer. 2015;15:623.

54. Kim YC, Guan KL. mTOR: a pharmacologic target for autophagy regulation. J Clin Invest. 2015;125(1):25-32.
55. Liu Y, Tong L, Luo Y, Li X, Chen G, Wang Y. Resveratrol inhibits the proliferation and induces the apoptosis in ovarian cancer cells via inhibiting glycolysis and targeting AMPK/mTOR signaling pathway. J Cell Biochem. 2018;119(7):6162-72.

56. Xu K, Liu XF, Ke ZQ, Yao Q, Guo S, Liu C. Resveratrol modulates apoptosis and autophagy induced by high glucose and palmitate in cardiac cells. Cell Physiol Biochem. 2018:46(5):2031-40.

57. Gan PP, Zhou YY, Zhong MZ, Peng Y, Li L, Li JH. Endoplasmic reticulum stress promotes autophagy and apoptosis and reduces chemotherapy resistance in mutant p53 lung Cancer cells. Cell Physiol Biochem. 2017:44(1):133-51.

58. Deegan S, Saveljeva S, Gorman AM, Samali A. Stress-induced selfcannibalism: on the regulation of autophagy by endoplasmic reticulum stress. Cell Mol Life Sci. 2013;70(14):2425-41.

59. Dominguez-Martin E, Ongay-Larios L, Kawasaki L, Vincent O, Coello G, Coria $R$, et al. IreA controls endoplasmic reticulum stress-induced autophagy and survival through homeostasis recovery. Mol Cell Biol. 2018:38(13):e00054-18.

60. Wang J, Qi Q, Zhou W, Feng Z, Huang B, Chen A, et al. Inhibition of glioma growth by flavokawain $B$ is mediated through endoplasmic reticulum stress induced autophagy. Autophagy. 2018;14(11):2007-22.

61. Shimizu S, Kanaseki T, Mizushima N, Mizuta T, Arakawa-Kobayashi S, Thompson CB, et al. Role of Bcl-2 family proteins in a non-apoptotic programmed cell death dependent on autophagy genes. Nat Cell Biol. 2004:6(12):1221-8

62. Yoshida GJ. Therapeutic strategies of drug repositioning targeting autophagy to induce cancer cell death: from pathophysiology to treatment. J Hematol Oncol. 2017;10(1):67.

63. Shimizu S, Yoshida T, Tsujioka M, Arakawa S. Autophagic cell death and cancer. Int J Mol Sci. 2014;15(2):3145-53.
Ready to submit your research? Choose BMC and benefit from:

- fast, convenient online submission

- thorough peer review by experienced researchers in your field

- rapid publication on acceptance

- support for research data, including large and complex data types

- gold Open Access which fosters wider collaboration and increased citations

- maximum visibility for your research: over $100 \mathrm{M}$ website views per year

At $\mathrm{BMC}$, research is always in progress.

Learn more biomedcentral.com/submissions 